\title{
Diseño de un plan de manejo forestal para el bosque de la Hacienda el Prado
}

\author{
Design of a forest management plan for the Hacienda el Prado forest
}

Desenho de um plano de manejo florestal para a floresta Hacienda el Prado

Carlos Andrés Álvarez Poveda ${ }^{1}$

caapfenix@hotmail.es

Henry Casamín Serrano ${ }^{2}$

leonora.casamin@hotmail.com

1Programa de Educación Continua de Sostenibilidad Empresarial y Reputación Social Corporativa, Escuela Politécnica del Ejército, Ecuador

${ }^{2}$ IASA, Escuela Politécnica del Ejército, Ecuador

Artículo recibido enero 2018, arbitrado febrero 2018 y publicado en mayo 2018

\section{RESUMEN}

Se realizó un plan de manejo forestal para el bosque de la Hacienda El Prado, con la finalidad de obtener la licencia de aprovechamiento forestal que permita aprovechar los recursos forestales del bosque de manera sostenible y sustentable. Primeramente se determinó el área de bosque, luego se realizó una valoración de los recursos forestales existentes y en base a esta información se llenaron los formularios del Plan Operativo de Aprovechamiento (POAP), para el manejo sustentable de los bosques andinos, requisitos necesarios para el trámite de obtención de la licencia de aprovechamiento forestal. El área del bosque de eucalipto encontrada en la hacienda El Prado fue de 113 ha, de las cuales 72,32 ha correspondieron a pendiente alta y 40,68 ha a pendiente leve. Con la información recolectada, se elaboró un plan operativo de aprovechamiento, en donde se detallan las tareas de aprovechamiento selectivo en el área previamente preparada y delimitada y los volúmenes de madera de eucalipto a ser cortada, aprovechada, utilizada y/o afectada, directamente; este plan deberá ser presentado en el Ministerio del Ambiente para la obtención de la licencia de aprovechamiento forestal.

Palabras clave: Área basal; Plan de manejo forestal; Sostenible; Sustentable

\begin{abstract}
A forest management plan was developed for the Hacienda El Prado forest, in order to obtain the forest exploitation license that allows the forest resources to be used in a sustainable and sustainable manner. Firstly, the forest area was determined, then an assessment of the existing forest resources was carried out and based on this information, the forms of the Operational Use Plan (POAP) were completed, for the sustainable management of Andean forests, necessary requirements for the procedure for obtaining the forest exploitation license. The eucalyptus forest area found at the El Prado hacienda was 113 ha, of which 72.32 ha corresponded to a high slope and 40.68 ha to a slight slope. With the information collected, an operational harvesting plan was prepared, detailing the selective harvesting tasks in the previously prepared and delimited area and the volumes of eucalyptus wood to be cut, harvested, used and / or affected, directly; This plan must be presented to the Ministry of the Environment to obtain the forest exploitation license.
\end{abstract}

Key words: Basal area; Forest management plan; Sustainable; Sustainable 


\section{RESUMO}

Um plano de manejo florestal foi desenvolvido para a floresta Hacienda El Prado, a fim de obter a licença de exploração florestal que permite que os recursos florestais sejam utilizados de maneira sustentável e sustentável. Primeiramente, foi determinada a área florestal, em seguida, foi realizada uma avaliação dos recursos florestais existentes e, com base nessas informações, foram concluídas as formas do Plano de Uso Operacional (POAP), para o manejo sustentável das florestas andinas, requisitos necessários para a procedimento para obtenção da licença de exploração florestal. A área de floresta de eucalipto encontrada na fazenda El Prado era de 113 ha, dos quais 72,32 ha correspondiam a uma inclinação alta e 40,68 ha a uma inclinação leve. Com as informações coletadas, foi elaborado um plano operacional de colheita, detalhando as tarefas de colheita seletiva na área previamente preparada e delimitada e os volumes de madeira de eucalipto a serem cortados, colhidos, usados e / ou afetados diretamente; Esse plano deve ser apresentado ao Ministério do Meio Ambiente para obter a licença de exploração florestal.

Palavras-chave: Área basal; Plano de manejo florestal; Sustentável; Sustentável

\section{INTRODUCCIÓN}

La Evaluación de los Recursos Forestales Mundiales (FAO, 2000), concluyó que la cobertura forestal mundial hasta el año 2000 fue de 3,9 mil millones de ha. La deforestación neta a nivel mundial se estimó en una tasa anual de aproximadamente 9 millones de ha, con una tasa de deforestación mundial bruta de 13,5 millones de ha y una tasa anual de 4,5 millones de ha de nuevas plantaciones sembradas, fue acentuadamente superior a las anteriores, y los rodales sembrados con éxito estimados en 3 millones de ha año-1 para todo el decenio de 1990, compensaron las pérdidas de los bosques naturales. Además la FAO (2000) concluyó que las iniciativas relacionadas con la ordenación sostenible de los bosques han estimulado a muchos países a desarrollar planes de manejo para sus bosques.

El estudio es llevado a cabo en la hacienda El Prado ubicada en el Ecuador, Provincia de Pichincha, Cantón Rumiñahui, Parroquia San Fernando. (Latitud 00 23' 20" S, Longitud: $78^{\circ} 24^{\prime} 44^{\prime \prime}$ ) ) a una altitud de 2748 m.s.n.m., una temperatura promedio de 13,96 grados centígrados y una precipitación media anual de 1332,72 mm; las lluvias en el sector tienen un régimen bimodal, el período lluvioso se presenta en octubre y mayo y además existe un déficit hídrico en julio y agosto (Arce, 2009). Corresponde a la formación vegetal bosque muy húmedo montano según Sierra (1999) y al ecotono de los pisos zoogeográficos temperado y alto andino ${ }^{1}$ (Pozo, Olmedo, y Espinoza, 2006).

Para finalizar el propósito de este estudio fue primeramente determinar el área de bosque, para luego aplicar una valoración de los recursos forestales existentes y en base a esta información se llenar los formularios del Plan Operativo de Aprovechamiento (POAP), para el manejo sustentable de los bosques andinos, requisitos necesarios para el trámite de obtención de la licencia de aprovechamiento forestal.

\section{MATERIALES Y MÉTODOS}

Los materiales que se utilizaron fueron los siguientes: hipsómetro, GPS, hojas de registro, cinta métrica, brújula, libreta de campo, materiales de oficina, 400 cartulinas de celulosa de 29 x $41 \mathrm{~cm}$, 4 litros de goma blanca de carpintero, 10 hilos dental blanco, 400 sobres $7 \times 4 \mathrm{~cm}, 2$ resma de papel bond de 80 g., sello de caucho numerados con tinta, herramientas (machetes, barras), fotografías, estacas, mapa digital, cámara digital. Implementos de oficina y programas informáticos como, BIODIVERSITY PRO ${ }^{\circledR}$, ORD $^{\circledR}, \quad$ EXCEL ${ }^{\circledR}, \quad$ AUTOCAD ${ }^{\circledR}, \quad$ INFOSTAT ${ }^{\circledR}$, BIOSTAT $^{\circledR}$, ARCGIS ${ }^{\circledR}$. Entrevista. Docente de la Carrera de Ciencias Agropecuarias IASA 1. 
Cálculo del área total de bosque mediante la utilización de un mapa base.

Para determinar el área de la zona de estudio se utilizó un mapa base de la hacienda El Prado a escala 1:5000 al cual se lo rasterizó y luego se procedió a vectorizarlo con el fin de obtener la altimetría de la zona en estudio.

\section{Área total de bosque}

Para poder georeferenciar la zona se procedió a tomar datos del perímetro del bosque con un receptor G.P.S.Garmin (con precisión de 3 metros), de igual manera se tomaron las coordenadas de cada parcela. Los datos tomados tanto de vectorización, como puntos del perímetro y parcelas se procesaron en el software ARCGIS ${ }^{\circledR}$. En este software se calculó área total del bosque en estudio así como también se determinaron las áreas con pendiente mayor y menor que $20 \%$.

\section{Determinación de abundancia, riqueza y diversidad de especies vegetales}

\section{Inventario forestal del bosque de Eucalyptus globulus}

Debido a las condiciones intrínsecas del bosque el inventario del bosque de Eucalyptus globulus de la hacienda El Prado se realizó utilizando un muestreo aleatorio estratificado. Se agrupó a las unidades con condiciones similares en dos estratos [pendiente leve, 40,68 ha. y pendiente alta, 72,32 ha de manera que las unidades de muestreo que estuvieron conformando el estrato fueron lo más homogéneas posible, y los estratos fueron heterogéneos entre sí. Para calcular el tamaño de la muestra se utilizaron los resultados de un inventario preliminar provenientes de 5 unidades de muestreo distribuidas equitativamente en los dos estratos.
Una vez establecido el tamaño de la muestra se ubicaron las parcelas en forma aleatoria por estratos en el mapa base y posteriormente se establecieron en el campo con la ayuda del GPS. En cada parcela de 0,1 ha $(20 \times 50 \mathrm{~m})$ se cuantificó el número total de árboles brinzales (árboles con DAP menor a $5 \mathrm{~cm}$ ) y el número total de árboles latizales (árboles con DAP entre 5 y $10 \mathrm{~cm}$ ). A todos los árboles con DAP mayor a $10 \mathrm{~cm}$ se midió el DAP, la altura total y la calidad del fuste. Posteriormente se determinó el área basal y el volumen total.

La información se analizó con estadística descriptiva, media, error estadístico, coeficiente de variación y diferentes técnicas de gráficos. También se realizaron gráficos de distribuciones diamétricas en función del número de árboles, del área basal y del volumen total. El software utilizado fue INFOSTAT $^{\circledR}$ (2012).

Para determinar las diferencias entre la cobertura arbórea presente en los diferentes estratos del bosque se realizaron análisis de modelos mixtos para comparar las variables cuantitativas diámetro promedio, altura total promedio, área basal promedio y volumen total promedio para los diferentes estratos. Además se realizaron pruebas de comparación de medias DMS al 5 \%.

\section{Determinación de riqueza, Abundancia y diversidad}

Para la determinación de riqueza, abundancia y diversidad de la vegetación menor se realizó una recolección de las especies para su posterior reconocimiento y descripción, con la ayuda de un experto del herbario nacional. Luego se procedió al conteo y etiquetado de las especies vegetales existentes en las 29 parcelas y las muestras botánicas fueron depositadas en el Herbario IASA (Facultad de Ciencias Agropecuarias de la ESPE). 


\section{Diversidad y abundancia}

Se determinó el número de las especies vegetales arbóreas y arbustivas diferentes a eucalipto en las 29 parcelas; con esta información se realizó una base de datos, en donde constan todas las especies encontradas, su abundancia y la presencia y/ o ausencia en las parcelas.

\section{Curvas rango de abundancia}

Las curvas de rango y abundancia se calcularon, dividiendo la abundancia de cada especie, para la abundancia total de todas las especies $(P i=n i / N)$. Con estos datos se sacó el valor de $P i$ el cual sirve para realizar un diagrama de líneas en 2D en el programa Excel ${ }^{\circledR}$.

\section{Índices de diversidad biológica}

Se estimaron los siguientes índices de biodiversidad: Berger-Parker Dominance (d), Simpsons Diversity (D), Simpsons Diversity (1/D), Hill'sNumber H1, Margaleff M, Mackintosh Distance (U), Mackintosh Diversity (D), Mackintosh Eveness (E) y Shannom-Winner, las fórmulas con las que se calcularon los índices se aprecian en el Cuadro 3 (Hill, 1973).

\section{Especies endémicas, raras y registros importantes}

\section{Similaridad de diversidad de especies}

Se realizó un análisis Bray-Curtis (BIODIVERSITY $\mathrm{PRO}^{\circledR}, 2012$ ), mediante el cual arrojó un dendrograma que permite conocer semejanzas y/o diferencias entre las 29 parcelas.

\section{Especies no registradas}

Son especies vegetales arbóreas y arbustivas que se encontraron en los caminos del bosque de la Hacienda el Prado.
Las especies fueron colectadas e identificadas mientras se realizó el inventario forestal, luego fueron colocadas en una base de datos, en el programa EXCEL ${ }^{\circledR}$ ya que estas no fueron procesadas estadísticamente, pero son de importancia debido a que se encuentran en gran número en muchas de zonas del bosque debido a que la región presenta una elevadísima riqueza biológica, con un alto grado de endemismo.

\section{Diseño de un plan de manejo forestal}

Se procedió a realizar un resumen ejecutivo que incluye una descripción breve de los objetivos, los aspectos metodológicos del inventario y censos, la propuesta silvícola, así como los aspectos generales del aprovechamiento. En cuanto la difusión de la información generada a través de la escritura de un boletín divulgativo, el proyecto elaborado se difundió mediante un boletín divulgativo.

\section{RESULTADOS Y DISCUSIÓN}

\section{Área total del bosque y parcelas}

El área total del bosque donde se realizó el estudio fue de 113 ha, de las cuales 40,68 ha; corresponden a la pendiente baja y 72,32 ha; a la pendiente alta.

\section{Riqueza, Abundancia y Diversidad de especies arbustivas y arbóreas}

\section{Inventario del bosque de Eucalyptus globulus de la Hacienda El Prado}

Se contabilizó un total de 3167 individuos de Eucalyptus globulus en $29000 \mathrm{~m}^{2}$ de bosque, correspondientes a las 29 parcelas de $1000 \mathrm{~m}^{2}$ (Cuadro 4). De estos 1419 (44,39\%) fueron brinzales, $397(12,42 \%)$ latizales y 1351 (42,33\%) individuos con un DAP mayor a $10 \mathrm{~cm}$, es decir árboles fustales. De los 1351 
individuos con DAP $>10 \mathrm{~cm}, 36$ están dentro de la calidad 1, 756 en la calidad 2 y 229 pertenecen a la calidad 3 .

\section{Distribución de diámetros a la altura de pecho y alturas en árboles con DAP > a $10 \mathrm{~cm}$}

En total se inventariaron 1351 árboles con DAP mayor a $10 \mathrm{~cm}$. El mayor porcentaje de los individuos 31,53\% estuvieron ubicados en la clase diamétrica 20 a $30 \mathrm{~cm}$. Solamente el 0,07 \% tuvieron fustes con diámetros entre 90 a $100 \mathrm{~cm}$. El DAP promedio fue $29,61 \mathrm{~cm}$.

\section{Distribución del área basal y volumen total por clases diamétricas en árboles de eucalipto presentes en las 29 parcelas}

El área basal total encontrada en los 1351 individuos con DAP > a $10 \mathrm{~cm}$ fue de 115,67 $\mathrm{m}^{2}$ (38.88 $\mathrm{m}^{2}$ ha-1). El mayor porcentaje del área basal $(21,02 \%)$ se encontró en individuos con DAP comprendido entre 30 a $40 \mathrm{~cm}$, mientras que el menor porcentaje de área basal $(0,67 \%)$ se encontró en árboles con DAP entre 90 a $100 \mathrm{~cm}$.

\section{Distribución de diámetros a la altura de pecho}

La distribución diamétrica del eucalipto en las parcelas con pendiente leve y alta fue muy variable. Las parcelas con pendiente alta presentaron la mayor cantidad de árboles $(177,14)$ en la clase entre 20 y $30 \mathrm{~cm}$ y la menor cantidad de individuos $(0,71)$ se encontraron en la clase diamétrica entre $90 \mathrm{y}$ $100 \mathrm{~cm}$. Las parcelas con pendiente leve tuvieron mayor cantidad de individuos $(79,29)$ en la clase entre 20 y $30 \mathrm{~cm}$ y la menor cantidad de individuos $(5,05)$ en la clase diamétrica de 80 a $90 \mathrm{~cm}$.

\section{Diversidad y abundancia distinta al Eucalipto}

Se registraron 1845 especies vegetales (Cuadro 9), la abundancia estuvo distribuida en 31 especies, pertenecientes a 24 diferentes familias botánicas (Cuadro 10), de las cuales la más abundante fue la familia Asteraceae con 250 individuos y las más ricas fueron las familias Astereraceae y Rosaceae con 3 especies, respectivamente.

\section{Curvas de rango de abundancia}

Las familias que presentaron mayor rango de abundancia son Euphorbiaceae y Piperaceae. En los rangos de abundancia por especie Phyllanthus salviifolius, Piper sp., Rubus adenotrichos son las especies con mayor rango.

\section{Índices de diversidad biológica}

Un indicador puede definirse como "una medida basada en datos verificables que transmite información más allá de sí mismo". Esto significa que los indicadores están subordinados al propósito, es decir, la interpretación o el significado que se atribuye a los datos depende del propósito o del tema de interés.

Los índices de diversidad incorporan en un solo valor a la riqueza específica y a la equitabilidad. En algunos casos un valor dado de un índice de diversidad puede provenir de distintas combinaciones de riqueza específica y equitabilidad.

\section{Especies endémicas, raras y registros importantes}

\section{Especies endémicas}

La región presenta una elevadísima riqueza biológica, con un alto grado de 
endemismo, se registraron las siguientes especies endémicas de importancia Oreopanax ecuadorensis, Prunus serotina, Phyllanthus salviifolius, Baccharis latifolia, Monnina obtusifolia, Dalea coerulea, Salvia quitensis. Hay otras especies que no fueron registradas dentro de las 29 parcelas de estudio, pero se encuentran distribuidas en grandes zonas del bosque, y caminos como Biden striplinervia, Hypochaeris sessiliflora, Brugmansia sanguínea, Datura stramonium, Solanum nigrescens, Mimosa albida, Epidendrum jamiesonis, Castilleja arvensis, Sida rhombifolia. (Añazco, 2000)

Durante el inventario forestal realizado en las 29 parcelas, se encontraron 2 especies abundantes (Phyllanthus salviifolius, Piper sp), Cabe indicar que normalmente los criterios de clasificación en grados de abundancia de las especies se basan en el número de individuos registrados en las especies y obedecen al libre albedrio de los autores. Para evitar algún sesgo en ese sentido, se calcularon los rangos de abundancia (Pi) de las especies para calificarlas según Pozo (2013).

Abundantes (A): si tienen un valor $P i$ igual o mayor a 0,1 y la abundancia sobrepasa los 150 individuos;

Comunes (C): si su valor Pi oscila entre 0,05 y 0,09 y la abundancia sobrepasa los 60 individuos;

Frecuentes (F): si su Pi oscila entre 0,01 y 0,04 y su abundancia esta entre 15 y 50 individuo;

Rara (R): si su Pi es menor que 0,01 y su abundancia es menor a 15 individuos. Este criterio de clasificación es muy particular para el área de estudio y dependerá su aplicación según el tamaño del área monitoreada.

\section{Plan de manejo forestal}

Previo a la emisión de la Licencia para la explotación, con la información recolectada, se elaboró un plan de manejo forestal en base al plan operativo de aprovechamiento, en donde se detallan las tareas de aprovechamiento selectivo en el área previamente preparada y delimitada y los volúmenes de madera de eucalipto a ser cortada, aprovechada, utilizada y/o afectada, directamente.

Con este plan se determinó los volúmenes de la madera de eucalipto a ser cortada, aprovechada, utilizada y/o afectada, directamente.

\section{CONCLUSIONES}

El área de bosque de eucalipto encontrada en la hacienda El prado fue de 113 ha, de las cuales 72,32 ha corresponden a la pendiente alta y 40,68 ha a la pendiente leve. En las 29 parcelas inventariadas (2,9 ha) se encontraron un total de 3169 árboles (1351 ha $^{-1}$ ) de los cuales 1419 fueron brinzales, 397 latizales y 1351 fustales; del total de los fustales 367 tuvieron calidad 1; 755 calidad 2 y 229 calidad 3.

El área basal promedio encontrada fue de $115,67 \mathrm{~m}^{2} \mathrm{ha}^{-1} \mathrm{y}$ el volumen total promedio fue de 1532,22 $\mathrm{m}^{3} \mathrm{ha}^{-1}$. El número de árboles, fue superior en zonas con pendiente alta, que en zonas con pendiente baja, mientras que DAP, altura total, área basal y volumen total fue mayor en zonas con pendiente leve, que en las zonas con pendiente alta.

Los volúmenes de corta anual permisible inicial y final para las zonas con pendiente alta fueron $362,44 \mathrm{~m}^{3} \mathrm{ha}^{-1}$, y $404,10 \mathrm{~m}^{3} \mathrm{ha}^{-1}$, respectivamente; mientras que para zonas con pendiente leve fueron 193,36 $\mathrm{m}^{3} \mathrm{ha}^{-1} \mathrm{y}$ $206,42 \mathrm{~m}^{3}$ ha $^{-1}$, respectivamente.

En el bosque de la hacienda El Prado se registraron 1845 individuos, pertenecientes a 31 especies, y a 24 diferentes familias botánicas, de las cuales la más abundante fue la familia Asteraceae con 250 individuos y las más ricas fueron las familias Astereraceae y Rosaceae con 3 especies respectivamente. 
Se registraron las siguientes especies de importancia Oreopanax ecuadorensis, Prunus serotina, Phyllanthus salviifolius, Baccharis latifolia, Monnina obtusifolia, Dalea coerulea, Salvia quitensis.

Algunas recomendaciones a considerar es importantes determinar los criterios generales sobre los cuales se deberá planificar y ejecutar el manejo forestal sustentable. Se debe implementar un plan de prevención y control de incendios forestales con el fin de disminuir las alteraciones del ecosistema natural. Determinar las áreas de recuperación con pendientes superiores al $100 \%$, para que sean destinadas exclusivamente a la restauración a través del manejo de la regeneración natural o enriquecimiento con especies nativas. Zonificar las áreas donde se va a aprovechar la madera. Determinar las zonas de protección permanente en el bosque.

\section{REFERENCIAS}

Añazco, M. (2000). Selección de especies y manejo de semillas. Impresión Rispergraf. Quito- EC.58p

Arce, M. (2009). Normal climática y distribución de la precipitación de la Hacienda El Prado-Iasa.
ArcGIS. (s.f.). ArcGIS Resource Centers.Consultado 1 de Enero del 2012, de

http://resources.esri.com/arcgisserver/a df/dotnet/index.cfm?fa=home

Biodiversity Pro Version 2 (en línea) The Natural History Museum and The Association for Marine Science.N .Y 1997.Consultado 1 de Enero de 2012. Disponible en: http://biodiversitypro.software.informer.com/2.0/

Boletín Técnico 8, Serie Zoológica 4: 126-128

Bravo, F. (2001). Forest structure and diameter growth in Maritime pine in a Mediterranean area. En: von Gadow, K., Saborowski, J. (Ed.), IUFRO Conference on continuous cover forestry, Göttingen, pp. 101-110

Fao. (2000). El Estado mundial de la pesca y la acuicultura, 2000. Food \& Agriculture Org

Pozo W.E., I. Olmedo, y S. Espinoza. (2006). Diversidad rodentológica en remanentes de bosque nativo y cercas vivas de la hacienda El Prado, serranía ecuatoriana. Boletín Técnico 6, Serie Zoológica 2: 33-44

Sierra, R. (1999). Propuesta preliminar de un sistema de clasificación de vegetación para el Ecuador Continental. Proyecto INEFAN/GEF-BIRF y EcoCiencia. Quito Ecuador 